\title{
CNO abundances in giants of the peculiar globular cluster NGC 1851
}

\author{
G. Tautvaišienè ${ }^{1}$ A. Drazdauskas, ${ }^{1}$ C. Lardo, ${ }^{2}$ S. L. Martell, ${ }^{3}$ \\ E. Pancino, ${ }^{4,5}$ E. Stonkutè, ${ }^{1}$ and Gaia-ESO Consortium \\ ${ }^{1}$ Institute of Theoretical Physics and Astronomy, Vilnius University, Gostauto 12, \\ 01108, Vilnius, Lithuania, email: grazina.tautvaisiene@tfai.vu. It \\ ${ }^{2}$ Astrophysics Research Institute, Liverpool John Moores University, 146 Brownlow Hill, \\ Liverpool L3 5RF, United Kingdom \\ ${ }^{3}$ School of Physics, University of New South Wales, NSW 2052, Sydney, Australia \\ ${ }^{4}$ INAF-Osservatorio Astronomico di Bologna, Via Ranzani 1, 40127, Bologna, Italy \\ ${ }^{5}$ ASI Science Data Center, Via del Politecnico SNC, 00133, Roma, Italy
}

\begin{abstract}
We provide CNO and Fe abundance investigations for a sample of up to 45 NGC 1851 giants. High-resolution spectra were obtained with the VLT UVES spectrograph in the framework of the Gaia-ESO Survey. The stars in our sample can be separated into two groups with a difference of 0.1 dex in the mean metallicity, 0.3 dex in the mean $\mathrm{C} / \mathrm{N}$, and no significant difference in the mean values of $\mathrm{C}+\mathrm{N}+\mathrm{O}$.
\end{abstract}

Keywords. Stars: abundances, stars: evolution, globular clusters: individual (NGC 1851)

\section{Introduction}

It has been long suspected that NGC 1851 is not chemically homogeneous (e.g., Walker 1992). By now, it is clear that NGC 1851 has two distinct subgiant branches, however explanations for their origin so far lack consensus. Some authors explain them by two generations of stars, the first being primordial, while the second one being born from the ejecta of a fraction of the stars of the first population (e.g., see the review by Gratton et al. 2012). There are suggestions that NGC 1851 originated by merging of two globular clusters (e.g. Campbell et al. 2012), or is a naked nucleus of a captured and disrupted dwarf galaxy (e.g. Bekki \& Yong 2012; Marino et al. 2014). van den Bergh (1996) and later Carretta et al. (2010) joined two hyphoteses into one and suggested that NGC 1851 may have been formed by the merger between parental globulars that were once located within a dwarf spheroidal galaxy. The CNO content of NGC 1851 stars is a crucial observational constrain to test the proposed globular clusters formation scenarios. In the following, we present $\mathrm{CNO}$ abundances and metallicity determinations for a sample of up to 45 RGB stars in NGC 1851 from high-resolution spectral observations and archival data in the framework of the Gaia-ESO Survey.

\section{Observations and method of analysis}

Observations were conducted with the FLAMES multi-fiber facility on the ESO VLT telescope. Spectra of high-resolving power $(R \approx 47000)$ were obtained in a wavelength interval of $4700-6840 \AA$ with a gap of about $50 \AA$ in the centre. Signal-to-noise ratios in the observed spectra range from 40 to 180 depending on the stellar brightness.

A differential model atmosphere technique which was applied for the determination of the atmospheric parameters is described by Smiljanic et al. (2014). Approximate uncertainties of the main atmospheric parameters are $55 \mathrm{~K}, 0.13 \mathrm{dex}$, and 0.07 dex for 


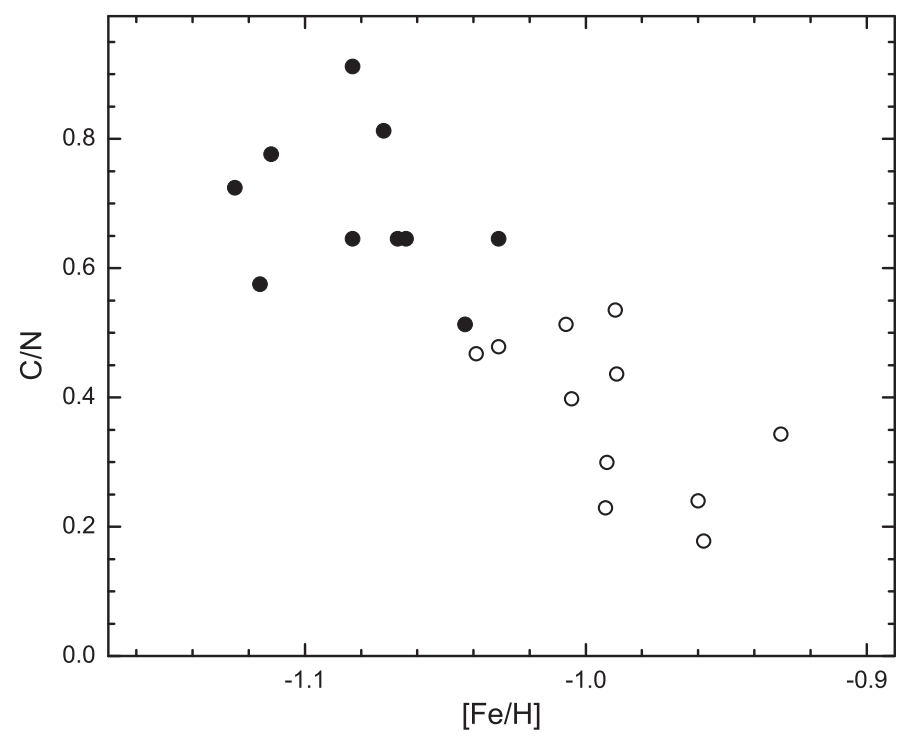

Figure 1. $\mathrm{C} / \mathrm{N}$ ratios in respect to $[\mathrm{Fe} / \mathrm{H}]$ for the NGC 1851 metal-rich (open circles) and metal-poor (filled circles) stars.

$T_{\text {eff }}, \log \mathrm{g}$, and $[\mathrm{Fe} / \mathrm{H}]$, respectively. The $\mathrm{C}_{2}$ Swan $(1,0)$ band head at $5135 \AA$ and $\mathrm{C}_{2}$ Swan $(0,1)$ band head at $5635.5 \AA$ were investigated in order to determine the carbon abundance. The interval 6470-6490 $\AA$ containing CN bands was used for the nitrogen abundance analysis. The oxygen abundance was determined from the forbidden $[\mathrm{O} \mathrm{I}]$ line at $6300.31 \AA$. More details about the analysis can be found in Tautvaišienè et al. (2015).

\section{Results}

We were able to separate NGC 1851 stars between metal-rich and metal-poor ones according to their metallicity and CNO content. Metal-rich and metal-poor stars have averaged metallicities $-0.99 \pm 0.03$ and $-1.08 \pm 0.03$, respectively. Fig. 1 shows how the sampled stars are located in the $\mathrm{C} / \mathrm{N}$ vs. $[\mathrm{Fe} / \mathrm{H}]$ plane.

The metal-rich and metal-poor subsamples have distinct abundances of nitrogen and consequently different values of $\mathrm{C} / \mathrm{N}$ ratios. The mean value of the $\mathrm{C} / \mathrm{N}$ ratio in metal rich stars is $0.40 \pm 0.14$, and in metal-poor stars is $0.68 \pm 0.12$. We will present the full analysis of this interesting cluster in a forthcoming paper.

Acknowledgements. Based on data products from observations made with ESO Telescopes at the La Silla Paranal Observatory under programme ID 188.B-3002. This work was supported by the grant from the Research Council of Lithuania (MIP-082/2015).

\section{References}

Bekki, K. \& Yong, D. 2012, MNRAS, 419, 2063

Campbell, S. W., Yong, D., Wylie-de Boer, E. C. et al. 2012, ApJ (Letters), 761, L2

Carretta, E., Gratton, R. G., Lucatello, S. et al. 2010, ApJ (Letters), 722, L1

Gratton, R., Carretta, E., \& Bragaglia, A. 2012, A\&ARv, 20, 50

Marino, A. F., Milone, A. P., \& Yong, D. et al. 2014, MNRAS, 442, 3044

Smiljanic, R., Korn, A. J., Bergemann, M. et al. 2014, A\& $A, 570,122$

Tautvaišienè, G., Drazdauskas, A., Mikolaitis, Š. et al. 2015, A\&AA, 573, 55

Walker, A. R. 1992, PASP, 104, 1063

van den Bergh, S. 1996, ApJ, 471, L31 